\title{
Effect of Sacubitril/Valsartan on VEGF, VEGFR-1 and Left Ventricular Remodeling in Patients with Heart Failure with Decreased Ejection Fraction
}

\author{
Xia Han*1, Menghai $\mathrm{Wu}^{2}$, Huiqin $\mathrm{Qi}^{1}$ and Wenting Liu ${ }^{1}$ \\ ${ }^{1}$ Department of Cardiology, Jinan People's Hospital Affiliated to Shandong First Medical University, China \\ ${ }^{2}$ Department of Neurology, Jinan People's Hospital Affiliated to Shandong First Medical University, China
}

*Corresponding author: Xia Han, Department of Cardiology, Jinan People's Hospital Affiliated to Shandong First Medical

University, Laiwu 271199, China

\begin{abstract}
ARTICLE INFO
Received: 幽 January 30, 2020

Published: 㓞 February 10, 2020

Citation: Xia Han, Menghai Wu, Huiqin Qi, Wenting Liu. Effect of Sacubitril/Valsartan on VEGF, VEGFR-1 and Left Ventricular Remodeling in Patients with Heart Failure with Decreased Ejection Fraction. Biomed J Sci \& Tech Res 25(3)-2020. BJSTR. MS.ID.004203.
\end{abstract}

Keywords: Myocardial Infarction; Heart Failure; Sacubitril/Valsartan; VEGF; VEGFR-1

\section{ABSTRACT}

Background: Sacubitril/valsartan has been shown to reduce mortality and reduce hospitalization in patients with heart failure with reduced ejection fraction (HFrEF), but it has not been reported for endothelial function and left ventricular remodeling in patients with heart failure. Endothelial cell dysfunction is involved in the progression of heart failure.

Hypothesis: So, we hypothesized that Sacubitril/valsartan improves left ventricular remodeling and prognosis in patients with heart failure by affecting VEGF and VEGFR-1.

Methods: From October 2018 to May 2019, 63 patients with heart failure who had been hospitalized in the Department of Cardiology of Jinan People's Hospital were selected. According to the treatment plan, they were divided into control group (37 cases) and observation group (26 cases). Patients in the control group underwent routine anti-heart failure treatment. Patients in the observation group were replaced with angiotensin-converting enzyme inhibitor (ACEI)/angiotensin II receptor antagonist (ARB) in the conventional anti-heart failure treatment regimen. Valsartan; both groups of patients were treated for 3 months. The levels of NT-proBNP, VEGF, VEGFR-1 and cardiac structural parameters were compared before and after treatment in the two groups.

Results: There were significant differences in VEGF, VEGFR-1 and pro-BNP between the two groups after T treatment.

Conclusion: After 3 months of treatment with Sacubitril/valsartan, patients with heart failure with reduced ejection fraction improved left ventricular systolic function, increased plasma VEGF levels, and decreased NT-proBNP and VEGF-1R levels.

\section{Introduction}

Percutaneous Coronary Intervention (PCI) completely altered the management and treatment of acute Myocardial Infarction (MI) $[1,2]$. It is a reperfusion strategy for the entire developed country, with only 90,000 operations performed annually in the United States $[3,4]$. The introduction of PCI and adjuvant therapy have reduced hospital mortality after acute Myocardial Infarction from $20 \%$ in the late 1980 s to about $5 \%-7 \%$ in the modern series $[5,6]$. Heart failure after Myocardial Infarction remains a major driver of coronary heart disease in patients with advanced morbidity, mortality, and medical costs. There is therefore an urgent need to explore new therapies for patients with reduced ejection fraction. A new type of drug for the treatment of heart failure, Sacubitril/ valsartan, has been developed, a salt complex crystal composed of Using an open-ended test, the observation group and the control group were randomly divided according to the random number table method.

Patients in the control group were treated with conventional anti-heart failure, including rest, salt restriction and diuretics, digitalis preparations, vasodilators, beta-blockers, AngiotensinConverting Enzyme Inhibitors (ACEI)/angiotens Receptor II 
receptor antagonist (ARB), spironolactone treatment, etc., and adjust the treatment according to the primary disease; patients in the observation group replaced the ACEI/ARB in the conventional anti-heart failure treatment plan with Sacubitril/valsartan (Beijing) Novartis Pharmaceutical Co., Ltd. produces, Chinese medicine quasi-word H20170344), starting dose 25 mg / time, 2 times / d, then gradually increase the dose, the maximum dose is $400 \mathrm{mg} / \mathrm{d}$, in order to overlap the risk of angioedema caused by ACEI To the lowest, before the start of the use of Using an open-ended test, the observation group and the control group were randomly divided according to the random number table method.

Patients in the control group were treated with conventional anti-heart failure, including rest, salt restriction and diuretics, digitalis preparations, vasodilators, beta-blockers, AngiotensinConverting Enzyme Inhibitors (ACEI)/Angiotens Receptor II Receptor Antagonist (ARB), spironolactone treatment, etc., and adjust the treatment according to the primary disease; patients in the observation group replaced the ACEI/ARB in the conventional anti-heart failure treatment plan with Sacubitril/valsartan (Beijing) Novartis Pharmaceutical Co., Ltd. produces, Chinese medicine quasi-word H20170344), starting dose 25 mg / time, 2 times / d, then gradually increase the dose, the maximum dose is $400 \mathrm{mg} /$ $\mathrm{d}$, in order to overlap the risk of angioedema caused by ACEI To the lowest, before the start of the use of Sacubitril/valsartan ACEI should be given at least $36 \mathrm{~h}$ for drug elution, the rest of the treatment with the control group. Both groups of patients were treated continuously for 3 months.

Combination therapy with ACE inhibitor (or ARB) and Sacubitril/valsartan is strictly prohibited. Two groups of patients underwent routine clinical examination before treatment with anti-heart failure, 12-lead electrocardiogram (ECG), transthoracic echocardiography (TTE) and Doppler assessment. The same measurement was repeated at 3 months. Cardiac function assessment was performed using NYHA classification. / valsartan, ACEI should be given at least $36 \mathrm{~h}$ for drug elution, the rest of the treatment with the control group. Both groups of patients were treated continuously for 3 months. Combination therapy with ACE inhibitor (or ARB) and Sacubitril/valsartan is strictly prohibited. Two groups of patients underwent routine clinical examination before treatment with anti-heart failure, 12-lead electrocardiogram (ECG), transthoracic echocardiography (TTE) and Doppler assessment. The same measurement was repeated at 3 months. Cardiac function assessment was performed using NYHA classification.and valsartan (LCZ696) in a 1:1 molar ratio [7] , PARADIGM-HF [ARNI vs. ACEI (a prospective comparison of angiotensin-converting enzyme inhibition to determine the overall mortality and morbidity of heart failure) study] is a phase III, randomized, double-blind trial evaluation. The safety and efficacy [8] of LCZ 696 and enalapril in patients with chronic symptomatic HFREF can reduce all-cause mortality in patients with chronic HFREF by $16 \%$, cardiovascular mortality by $20 \%$, and HF hospitalization by $21 \%$. However, in this landmark trial, the serum markers VEGF, VEGFR-1 and endothelial cell-controlled signaling pathways that do not clearly determine. Endothelial cell-controlled signaling pathways play a crucial homeostatic role in cardiac tissue and disorders of these pathways can lead to poor myocardial remodeling and dysfunction in heart failure, suggesting a poor prognosis in these patients.

Vascular Endothelial Growth Factor (VEGF) is a plateletderived growth factor supergene family secreted by Endothelial Cells and plays a central role in regulating angiogenesis and lymphangiogenesis. VEGF-A is a major angiogenic factor that binds to two Tyrosine Kinase (TK) receptors, VEGFR-1 (Flt-1) and VEGFR-2 (KDR/Flk-1), and regulates proliferation of endothelial cells. , migration, vascular permeability, secretion and other small molecule proteins or peptides. Our study aimed to evaluate the effect of Sacubitril/valsartan on endothelial cell function (VEGF/ VEGFR-1) and to further provide new therapeutic targets and clinical markers for heart failure.

\section{Methods}

The study was approved by our Institutional Committee on Human Research. No extramural funding was employed to support this work. The authors are solely responsible for study design and conduct, study analyses, drafting and editing of the paper, as well as its final editorial content.

\section{Patient Selection}

From June 2017 to September 2018, patients with heart failure who had a reduced ejection fraction in the Department of Cardiology of Jinan City People's Hospital.

Standard Constrain: The patient is at least 18 years of age, with New York Heart Association (NYHA) II, III or IV symptoms and an ejection fraction of no more than $40 \%$. The patient was asked to be hospitalized for heart failure in the past 12 months. Consider patients who do not take any ACE inhibitors or ARB, or who take a steady dose of beta blockers, ACE inhibitors or ARB for at least 4 weeks.

Exclusion Criteria: Including symptomatic hypotension, systolic blood pressure $<100 \mathrm{mmHg}$, estimated glomerular filtration rate (eGFR) below body surface area $30 \mathrm{~mL} / \mathrm{min} /$ $1.73 \mathrm{~m} 2$, serum potassium level $>5.2 \mathrm{mmol} / \mathrm{L}$ during screening, history of angioedema Or unacceptable side effects occur with ACE inhibitors or ARB treatment. Other exclusion criteria were as follows: correctable valvular disease; $<3$ months of acute coronary syndrome; recent coronary revascularization within the last 3 months, or planned revascularization. According to previous studies, an (absolute) improvement in LVEF $\geq 5 \%$ is considered to be an important response to shakuba/valsartan $[9,10]$.

\section{Study Procedures}

Using an open-ended test, the observation group and the control group were randomly divided according to the random 
number table method. Patients in the control group were treated with conventional anti-heart failure, including rest, salt restriction and diuretics, digitalis preparations, vasodilators, beta-blockers, Angiotensin-Converting Enzyme Inhibitors (ACEI)/Angiotens Receptor II Receptor Antagonist (ARB), spironolactone treatment, etc., and adjust the treatment according to the primary disease; patients in the observation group replaced the ACEI/ARB in the conventional anti-heart failure treatment plan with Shakuba / valsartan (Beijing Novartis Pharmaceutical Co., Ltd. produces, Chinese medicine quasi-word H20170344), starting dose $25 \mathrm{mg} /$ time, 2 times / d, then gradually increase the dose, the maximum dose is $400 \mathrm{mg} / \mathrm{d}$, in order to overlap the risk of angioedema caused by ACEI To the lowest, before the start of the use of Sacubitril/ valsartan, ACEI should be given at least $36 \mathrm{~h}$ for drug elution, the rest of the treatment with the control group. Both groups of patients were treated continuously for 3 months. Combination therapy with ACE inhibitor (or ARB) and Sacubitril/valsartan/valsartan is strictly prohibited. Two groups of patients underwent routine clinical examination before treatment with anti-heart failure, 12lead electrocardiogram (ECG), transthoracic echocardiography (TTE) and Doppler assessment. The same measurement was repeated at 3 months. Cardiac function assessment was performed using NYHA classification.

\section{Echocardiographic Measurements}

Vivid E9, GE Healthcare, (USA) was used to detect patients taking the left lateral position and synchronizing the ECG. Subjects underwent routine cardiac ultrasound by an experienced cardiac sonographer (without knowing the patient's condition), using an s3 ultrasound probe with a probe frequency of $2.5 \mathrm{MH}$ and measuring left ventricular structural parameters such as end-systolic and diastolic at a standard plane recommended by the American Society of Ultrasound. End diameter (LVIDs, LVIDd), posterior wall end-systolic and end-diastolic thickness (LVPwTs, LVPWTd), systolic and end-diastolic volume (LVESV, LVEDV), systolic function (LVEF), diastolic function (E/A, EDT, IVRT) Routine indicators, left and right ventricle and left and right ventricle (LA, LV) in the apical four-chamber view. All indicators were measured continuously for 3 cycles. All echocardiograms are read, reviewed, and diagnosed by trained experts. LVEF: Left ventricular end-diastolic volume (LVEDV), left ventricular end-systolic volume (LVESV), and left ventricular ejection fraction (LVEF) were measured using the Simpson method. LVEF $=($ LVEDV - LVESV $) /$ LVEDV $\times 100 \%$.

\section{Detection of VEGF and VEGFR-1}

The kit uses a double antibody one-step sandwich enzymelinked immunosorbent assay (ELISA). The coated microcapsules, which are pre-coated with Vascular Endothelial Growth Factor (VEGF) antibody and Vascular Endothelial Growth Factor receptor 1 (VEGFR-1) antibody, are sequentially added with specimens, standards, and HRP-labeled detection antibodies. Breed and wash thoroughly. Using the substrate TMB to develop color, TMB is converted to blue under the catalysis of peroxidase and converted to the final yellow color by the action of an acid. The color depth is positively correlated with VEGF and VEGFR-1 in the sample. The absorbance (OD value) was measured at $450 \mathrm{~nm}$ using a microplate reader to calculate the sample concentration.

\section{Statistical Analysis}

Data analysis was performed using SPSS 13.0 statistical software. The measurement data were expressed as mean $\pm \mathrm{SD}$. The $t$ test was used for comparison between groups. The paired $t$ test was used for comparison within the group. The $\chi 2$ test was used for the analysis of the count data test. The difference was statistically significant at $\mathrm{P}<0.05$. The skewed distribution data is converted to a normal distribution (represented by the median) by log logarithm.

\section{Results}

\section{Population}

All patients were divided into control group (37 cases) and observation group ( 26 cases) according to the treatment plan. There were no significant differences in age, height, body weight, HR, BSA, Cr and NT-proBNP between the two groups ( $\mathrm{P}>0.05$, Table 1$)$. There were no significant differences in left ventricular structural parameters (LVEF), VEGF and VEGFR-1 between the control group and the observation group ( $\mathrm{P}>0.05)$. (See Tables $1 \& 2$ ).

\section{Comparison of Indicators Between the two Groups after Treatment}

After treatment, the cardiac structural parameters (AO, LVIDs, LA) and lgNT-proBNP in the control group were larger than those in the observation group, and the systolic function index (LVEF) was lower than that in the observation group. The difference was statistically significant $(\mathrm{P}<0.05)$. The VEGF of the control group was significantly lower than that of the observation group, and the level of VEGF-1R was higher than that of the observation group, and the difference was statistically significant $(\mathrm{P}<0.05)$ (See Table 3).

\section{Comparison of Indicators in the two Groups after Treatment}

Comparison between the two groups after treatment: Compared with before treatment, the cardiac structure index and lgNT-proBNP were significantly decreased in the observation group, and the systolic function index was significantly increased, the difference was statistically significant $(\mathrm{P}<0.05)$, before treatment. There was no significant difference in RA between the observation group and the control group $(\mathrm{P}>0.05)$. There were statistical changes between the control group and other indicators except PWTs, RA, RV, VEGF and VEGF-1R. Academic significance $(\mathrm{P}<0.05)$ (See Table 4).

\section{Correlation Analysis between VEGF and IgNT-proBNP, VEGF-1 and lgNTproBNP}

The results showed that VEGF and lgNT-proBNP were positively correlated after treatment, with a coefficient of $0.534(\mathrm{P}<0.05)$ (Figure 1). VEGF-1 and lgNT-proBNP were also positively correlated after treatment, with a coefficient of 0.459. $(\mathrm{P}<0.05)$ (Figure 2). 


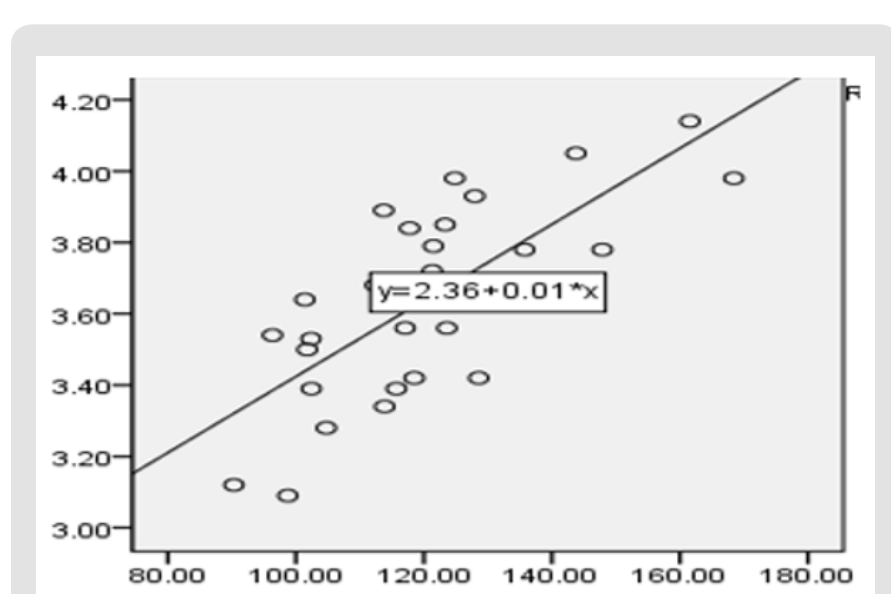

Figure 1: Linear relationship between VEGF and lgNTproBNP after treatment in the observation group.

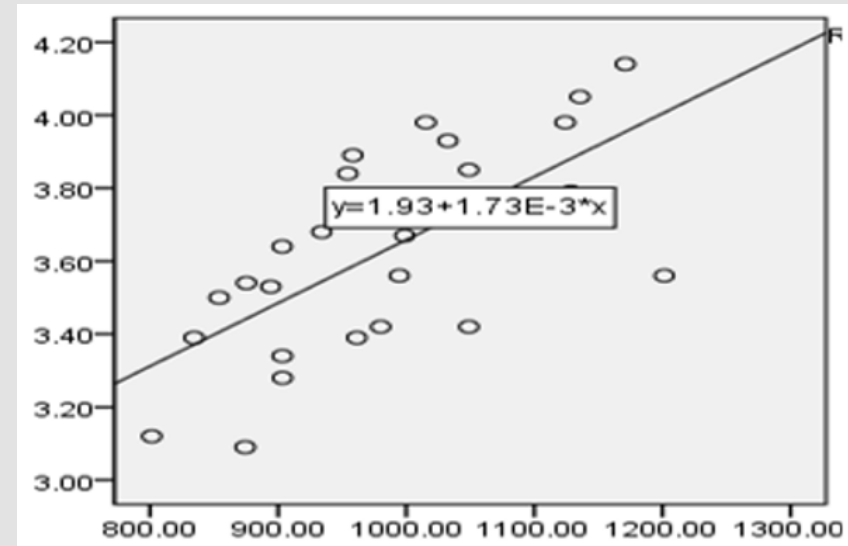

Figure 2: Linear relationship between VEGF-1 and lgNTproBNP after treatment in the observation group.

\section{Discussion}

Heart failure is defined as "a clinical syndrome caused by any structural or functional heart disease that impairs ventricular congestion or discharge of blood" [11]. This has been translated into several validated diagnostic criteria (eg, the Framingham standard [12] and the European Society of Cardiology standards [13]). Early studies of heart failure after Myocardial Infarction used clinical criteria such as the classification criteria of Killip and the New York Heart Association $[14,15]$. Although succinct, the Killip grading retains prognostic value in recent cohort studies (eg, the GRACE registry): hospitalization mortality in patients with Killip class I is $3 \%$, and in patients with class III is $20 \%$ [16]. In patients with PCI, a higher Killip grading is an independent predictor of hospitalization and 6-month mortality [17]. The development of echocardiography has improved the clinical HF score, which led to an objective measurement of ejection fraction and ventricular volume, which is an intrinsic part of HF diagnosis [18].

There are several overlapping mechanisms of $\mathrm{HF}$ after myocardial infarction. Early onset of $\mathrm{HF}$ in acute Myocardial Infarction is due to myocardial shock, myocardial necrosis, previous HF decompensation or a combination of acute mitral regurgitation due to papillary muscle dysfunction. Heart failure during hospitalization may also be due to any of the above reasons, accompanied by fluid or contrast overload, renal insufficiency or complications such as ventricular septal defect or cardiac tamponade. Late stages of heart failure reflect the consequences of simultaneous myocardial cell death and scar formation and ventricular remodeling. In the 1960s, Killip first used heart failure after Myocardial Infarction as a prognostic feature of poor prognosis. Heart failure is associated with extensive Myocardial Infarction infarction and multivessel disease, and impaired ventricular function leads to increased mortality [19]. Therefore, the treatment of heart failure after Myocardial Infarction remains a major challenge.

Sacubitril/valsartan is the first renin-angiotensinaldosterone system (RAAS) and enkephalinase double blocker, and its complementary mechanisms overlap. It is currently the "breakthrough" drug for the treatment of heart failure. The 2016 European Society of Cardiology Guidelines for the Diagnosis and Treatment of Acute and Chronic Heart Failure [20] is the first recommendation to use Sacubitril/valsartan for heart failure. Enkephalinase [8] is a membrane-bound endopeptidase that hydrolyzes the atria, brain and C-type natriuretic peptides and other endogenous vasodilators, such as adrenomedullin and bradykinin, to clear the above peptides. The main enzyme of the class. Thus, inhibition of enkephalinase leads to elevated levels of natriuretic peptides, with several potential benefits such as diuretic effects and vasodilation [7], dual inhibition of RAAS and enkephalinase translates into angiotensin II-mediated hypertrophy or fibrosis, as well as beneficial anti-proliferative and anti-hypertrophic effects. In general, natriuretic peptides are secreted by excessive blood volume and increased left ventricular filling pressure, which is a common feature in patients with heart failure. Therefore, Shakuba Qusarsartan helps regulate sodium and water balance, blood volume, arterial blood pressure and sympathetic inhibition. The PARADIGM HF study demonstrates the striking clinical benefits of LCZ696, primarily because of a significant reduction in the primary composite endpoint of cardiovascular death or HF hospitalization, and a reduced risk of death for any reason. Although the clinical benefits of shakuba/valsartan have been well documented to support the expected potential physiological mechanisms, other effects on tissue remodeling have not been well documented [21]. The mammalian genome encodes five VEGF family members, VEGF-A (also known as VEGF), placental growth factor (PlGF), VEGF-B, VEGF-C and VEGF-D, which regulate angiogenesis, angiogenesis and lymphangiogenesis [22, 23].

In particular, VEGF-A is critical for angiogenesis during early embryogenesis. Due to the formation of immature blood vessels, not only VEGF-A homozygous knockout mice but also heterozygous mice (VEGF-A +/-) showed an embryonic lethal phenotype, indicating that VEGF-A in embryos must be strictly controlled [24, 25]. Several VEGF-A subtypes were generated by alternative splic- 
ing. Among them, VEGF-A165 has the highest biological activity and has binding affinity to the co-receptor Neuropilin-1 (Nrp1). Recently, another spliced form of VEGF-A, VEGFxxxb, has been reported. VEGFxxxb has a lower affinity for the receptor and competes with VEGF-A, thereby negatively regulating angiogenesis [26].

In 1990, researchers isolated a gene encoding a novel Tyrosine Kinase (TK) receptor from human placenta. The TK receptor has seven immunoglobulin (Ig)-like domains in the extracellular region, while the TK domain has a 60 amino acid long kinase insert [27]. Based on structural similarity, we named it Fms-like TK-1 (Flt-1). In 1992, Flt-1 was shown to bind to VEGF/vascular permeability factor and is now known as VEGFR-1 [28]. Unlike VEGFR-2, VEGFR-1 has a high affinity for its ligand VEGF, and its affinity is about an order of magnitude higher than that of VEGFR-2 [29]. However, the kinase activity of VEGFR-1 is low, about one tenth of that of VEGFR-2. The VEGFR-1 gene produces two major proteins: the full-length receptor and sFlt-1 [30]. These facts suggest that VEGFR-1 may have a negative regulatory effect on angiogenesis in some cases. The study found that VEGFR-1 negatively regulates angiogenesis during early embryogenesis by capturing VEGF and decreasing the pro-angiogenic signal of VEGFR-2 [31].

The study found that LCZ696 acts in parallel with the NO-sGCcGMP pathway by activating the NP-pCG-cGMP signaling system, which increases diuresis and vasodilation. They also enhance myocardial relaxation through signaling pathways that are dependent on cardiac endothelial cells. Increasing cGMP production to reduce cardiac hypertrophy [32], inhibition of angiotensin receptors leading to inhibition of the renin-angiotensin-aldosterone system, which has been shown to be beneficial in heart failure, is consistent with our findings. Shakuba Qusarsartan improved left ventricular remodeling. The heart is a muscle pump composed of cardiomyocytes, Endothelial Cells (EC), fibroblasts, stem cells and inflammatory cells [33]. Cardiac tissue is a highly organized structure of cells and extracellular matrices with complex multi-directional communication between cells. All cells present in the myocardium secrete autocrine, juxtaposition and paracrine factors that regulate the function of neighboring cells. Intercellular communication plays a crucial role in the development of heart and normal heart function in adult organisms, and plays a crucial role in the pathophysiology of cardiac remodeling and heart failure development. In particular, factors secreted by cardiac microvascular EC play a crucial role in normal cardiac function and cardiac remodeling. During the pathophysiological development of heart failure, changes in hemodynamic and mechanical factors, as well as hypoxia, stimulate cardiomyocytes to release angiogenic growth factors, thereby inducing parallel growth of the supply vessels.

Vice versa, activated or dysfunctional Endothelial Cells may also affect the function of other types of cells in the heart. The study found that the pro-angiogenic effect of $\beta$-adrenergic receptor blockade in rat hearts was reduced by the administration of bait
Vascular Endothelial Growth Factor (VEGF) receptor (Ad-Flk) [34]. The positive nutrient effect of endothelial cell-derived nitric oxide (NO) leads to earlier relaxation episodes and longer diastolic phase [35] may also play a role in stimulating cardiac angiogenesis. Therefore, we found that the VEGF level in the observation group was significantly higher than that in the control group. Therefore, it is speculated that LCZ696 activates the NP-pCG-cGMP signaling system, activates Endothelial Cells to produce VEGF, stimulates angiogenesis and improves cardiomyocyte hypertrophy, and can pass endothelial cells. The paracrine function and intercellular signal series delay the fibrosis of cardiomyocytes; while the improvement of myocardial blood flow and left ventricular function directly stimulates the endothelial erbB receptor through autocrine, indirectly by increasing the expression of VEGF and angiopoietin-1 [36].

Cardiac Endothelial Cells not only respond to hemodynamic forces and paracrine signals of adjacent cells, but also actively participate in the cardiac remodeling process by stimulating the growth and contraction of cardiomyocytes or the production of extracellular matrix proteins in myoblasts. Furthermore, in response to appropriate signals, they may alter their phenotype and differentiate into extracellular matrix producing cells. Since cardiac angiogenesis plays a central role in the transition from adaptive cardiac hypertrophy to heart failure, Endothelial Cells and signaling mechanisms involved in the regulation or regulation of cardiac angiogenesis represent the potential to improve cardiac remodeling and prevent stress overload. A therapeutic target for arrhythmias.

\section{Author Contributions}

Han Xia designed this research. Wu Menghai collected samples and clinical data. Qi Huiqin and Liu Wenting carried out experiments. Wu Menghai analyzed the data, Han Xia wrote the paper.

\section{Acknowledgment}

The authors would like to thank Karin Fava for proofreading the article.

\section{Conflict of Interest Statement}

The authors declare that the research was conducted in the absence of any commercial or financial relationships that could be construed as a potential conflict of interest.

\section{References}

1. Nabel EG, Braunwald E (2012) A tale of coronary artery disease and myocardial infarction. N Engl J Med 366(1): 54-63.

2. Smilowitz NR, Feit F (2016) The History of Primary Angioplasty and Stenting for Acute Myocardial Infarction. Curr Cardiol Rep 18(1): 5.

3. Keeley EC, Boura JA, Grines CL (2003) Primary angioplasty versus intravenous thrombolytic therapy for acute myocardial infarction: a quantitative review of 23 randomised trials. Lancet 361(9351): 13-20.

4. Dehmer GJ, Weaver D, Roe MT, Milford Beland S, Fitzgerald S, et al. (2012) A contemporary view of diagnostic cardiac catheterization and percutaneous coronary intervention in the United States: a report from 
the CathPCI Registry of the National Cardiovascular Data Registry, 2010 through June 2011. J Am Coll Cardiol 60(20): 2017-2031.

5. Jernberg T, Johanson P, Held C, Svennblad B, Lindbäck J, et al. (2011) Association between adoption of evidence-based treatment and survival for patients with ST elevation myocardial infarction. JAMA 305(16): $1677-1684$

6. Puymirat E, Simon T, Steg PG, Schiele F, Guéret P, et al. (2012) Association of changes in clinical characteristics and management with improvement in survival among patients with ST-elevation myocardial infarction. JAMA 308(10): 998-1006.

7. Gu J, Noe A, Chandra P, Al Fayoumi S, Ligueros Saylan M, et al. (2010) Pharmacokinetics and pharmacodynamics of LCZ696, a novel dualacting angiotensin receptor-neprilysin inhibitor (ARNi). J Clin Pharmacol 50(4): 401-414.

8. McMurray JJ, Packer M, Desai AS, Gong J, Lefkowitz MP, et al. (2013) Dual angiotensin receptor and neprilysin inhibition as an alternative to angiotensin-converting enzyme inhibition in patients with chronic systolic heart failure: rationale for and design of the prospective comparison of ARNI with ACEI to Determine Impact on Global Mortality and morbidity in Heart Failure trial (PARADIGM-HF). Eur J Heart Fail 15(9): 1062-1073.

9. Pitzalis MV, Iacoviello M, Romito R, Guida P, De Tommasi E, et al. (2005) Ventricular asynchrony predicts a better outcome in patients with chronic heart failure receiving cardiac resynchronization therapy. J Am Coll Cardiol 45(1): 65-69.

10. Cintron G, Johnson G, Prancis G, Cobb F, Cohn JN (1993) Prognostic significance of serial changes in left ventricular ejection fraction in patients with congestive heart failure. The V HeFT VA Cooperative Studies Group Circulation 87(suppl): VI17-VI23.

11. Yancy CW, Jessup M, Bozkurt B, Butler J, Casey DE, et al. (2013) 2013 ACCF/AHA guideline for the management of heart failure: a report of the American College of Cardiology Foundation/American Heart Association Task Force on Practice Guidelines. J Am Coll Cardiol 62(16): e147-e239.

12. McKee PA, Castelli WP, McNamara PM, Kannel WB (1971) The natura history of congestive heart failure: the Framingham study. N Engl J Med 285(26): 1441-1446.

13. Swedberg K, Cleland J, Dargie H, Drexler H, Follath F, et al. (2005) Guidelines for the diagnosis and treatment of chronic heart failure: executive summary (update 2005): The Task Force for the Diagnosis and Treatment of Chronic Heart Failure of the European Society of Cardiology. Eur Heart J 26(11): 1115-1140.

14. Killip T, Kimball JT (1967) Treatment of myocardial infarction in a coronary care unit. A two year experience with 250 patients. Am J Cardiol 20(4): 457-464.

15. Spencer FA, Meyer TE, Goldberg RJ, Yarzebski J, Hatton M, et al. (1999) Twenty year trends (1975-1995) in the incidence, in-hospital and long-term death rates associated with heart failure complicating acute myocardial infarction: a community-wide perspective. J Am Coll Cardiol 34(5): 1378-1387.

16. Steg PG, Dabbous OH, Feldman LJ, Cohen Solal A, Aumont MC, et al. (2004) Determinants and prognostic impact of heart failure complicating acute coronary syndromes: observations from the Global Registry of Acute Coronary Events (GRACE) Circulation 109(4): 494-499.

17. DeGeare VS, Boura JA, Grines LL, O Neill WW, Grines CL (2001) Predictive value of the Killip classification in patients undergoing primary percutaneous coronary intervention for acute myocardial infarction. Am J Cardiol 87(9): 1035-1038.

18. Nicod P, Gilpin E, Dittrich H, Chappuis F, Ahnve S, et al. (1988) Influence on prognosis and morbidity of left ventricular ejection fraction with and without signs of left ventricular failure after acute myocardial infarction. Am J Cardiol 61(15): 1165-1171.
19. (1983) Risk stratification and survival after myocardial infarction. $\mathrm{N}$ Engl J Med 309(6): 331-336.

20. Ponikowski P, Voors AA, Anker SD, Bueno H, Cleland JGF, et al. (2016) 2016 ESC Guidelines for the diagnosis and treatment of acute and chronic heart failure: The Task Force for the diagnosis and treatment of acute and chronic heart failure of the European Society of Cardiology (ESC). Developed with the special contribution of the Heart Failure Association国HFA目of the ESC. Eur J Heart Fail 37(27): 2129-2200.

21. Fonarow GC, Hernandez AF, Solomon SD, Yancy CW (2016) Potential mortality reduction with optimal implementation of angiotensin receptor neprilysin inhibitor therapy in heart failure. JAMA Cardiol 1(6): 714-717.

22. Ferrara N, Kerbel RS (2005) Angiogenesis as a therapeutic target. Nature 438(7070): 967-974.

23. Shibuya M (2011) Involvement of Flt-1 (VEGFR-1) in cancer and preeclampsia. Proc Jpn Acad Ser B Phys Biol Sci 87(4): 167-178.

24. Ferrara N, Carver Moore K, Chen H, Dowd M, Lu L, et al. (1996) Heterozygous embryonic lethality induced by targeted inactivation of the VEGF gene. Nature 380(6573): 439-442.

25. Carmellet P, Ferreira V, Breier G, Pollefeyt S, Kleckens L, et al. (1996) Abnormal blood vessel development and lethality in embryos lacking a single VEGF allele. Nature 380(6573): 435-439.

26. Pritchard Jones RO, Dunn DB, Qiu Y, Varey AH, Orlando A, et al. (2007) Expression of VEGF(xxx)b, the inhibitory isoforms of VEGF, in malignant melanoma. Br J Cancer 97(2): 223-230.

27. Shibuya M, Yamaguchi S, Yamane A, Ikeda T, Tojo A, et al. (1990) Nucleotide sequence and expression of a novel human receptor-type tyrosine kinase gene (flt) closely related to the fms family. Oncogene 5(4): 519-524.

28. De Vries C, Escobedo JA, Ueno H, Houck K, Ferrara N, et al. (1992) The fms-like tyrosine kinase, a receptor for vascular endothelial growth factor. Science 255(5047): 989-991.

29. Sawano A, Takahashi T, Yamaguchi S, Aonuma T, Shibuya M (1996) Flt1 but not KDR/Flk-1 tyrosine kinase is a receptor for Placenta Growth Factor (PIGF), which is related to Vascular Endothelial Growth Factor (VEGF) Cell Growth Diff 7(2): 213-221.

30. Kendall RL, Thomas KA (1993) Inhibition of vascular endothelial cell growth factor activity by an endogenously encoded soluble receptor. Proc Natl Acad Sci 90(22): 10705-10709.

31. Hiratsuka S, Minowa O, Kuno J, Noda T, Shibuya M (1998) Flt-1 lacking the tyrosine kinase domain is sufficient for normal development and angiogenesis in mice. Proc Natl Acad Sci 95(16): 9349-9354.

32. Potter LR, Abbey Hosch S, Dickey DM (2006) Natriuretic peptides, their receptors, and cyclic guanosine monophosphate-dependent signaling functions. Endocr Rev 27(1): 47-72.

33. Kamo T, Akazawa H, Komuro I (2015) Cardiac nonmyocytes in the hub of cardiac hypertrophy. Circ Res 117(1): 89-98.

34. Rengo G, Cannavo A, Liccardo D, Zincarelli C, De Lucia C, et al. (2013) Vascular endothelial growth factor blockade prevents the beneficial effects of beta-blocker therapy on cardiac function, angiogenesis, and remodeling in heart failure. Circ Heart Fail 6(6): 1259-1267.

35. Gyurko R, Kuhlencordt P, Fishman MC, Huang PL (2000) Modulation of mouse cardiac function in vivo by eNOS and ANP. Am J Physiol Heart Circ Physiol 278(3): H971-981.

36. Gui C, Zeng ZY, Chen Q Luo YW, Li L, et al. (2018) Neuregulin-1 promotes myocardial angiogenesis in the rat model of diabetic cardiomyopathy. Cell Physiol Biochem 46(6): 2325-2334. 
ISSN: 2574-1241

DOI: $10.26717 /$ BJSTR.2020.25.004203

Xia Han. Biomed J Sci \& Tech Res

(c) (P) This work is licensed under Creative

Submission Link: https://biomedres.us/submit-manuscript.php

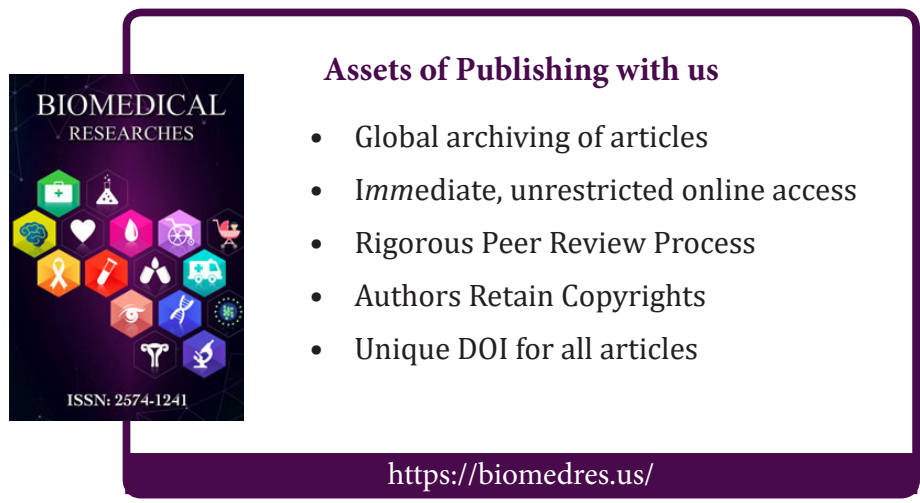

